\title{
Religión en el joven Hegel
}

\author{
Religion in the Young Hegel \\ Rafael ARAGÜÉS AliagA \\ Universidad de Heidelberg \\ rafael.aragues@gmail.com
}

DOI: http://dx.doi.org/10.15366/bp2016.12.004

Recibido: $29 / 11 / 2013$

Aprobado: $12 / 10 / 2016$

Resumen: El presente trabajo es un intento de profundizar en la motivación y el significado que tiene el problema de la religión en el joven Hegel. El artículo está centrado en los escritos de Berna (1793-1796) y sobre todo en los Fragmenten über Volksreligion und Christentum (1793-1794) y en Die Positivität der christlichen Religion (1794-1796). Cuál es la motivación fundamental que lleva a Hegel a reflexionar sobre religión, qué cariz tienen estas reflexiones y cuáles son sus ideas fundamentales son los puntos a aclarar en estas páginas.

Palabras clave: religión popular, positividad, el joven Hegel, Kant y Rousseau.

\begin{abstract}
The purpose of this paper is to research the concept of religion by the young Hegel. The article is based on the writings of Hegel's Berne period (1793-1796), specially on the Fragmenten über Volksreligion und Christentum (1793-1794) and on Die Positivität der christlichen Religion (1794-1796). On these pages we will ask ourselves about the reasons which make the young Hegel think about religion and the significance of religion in its early thought.
\end{abstract}

Keywords: folk religion, positivity, the young Hegel, Kant and Rousseau. 
La reflexión sobre la religión, sobre su sentido, relevancia y función dentro de una sociedad humana pensada a partir de principios racionales, es uno de los temas fundamentales del pensamiento de Hegel. Se trata, además, de un tema presente en todas las épocas de este autor, tanto en Berna, como en Frankfurt, Jena, Nürenberg y, finalmente, en Berlin. Y es, como se sabe, el tema central de los primeros escritos de Berna y del pensamiento del "joven" Hegel, escritos caracterizados en su día erróneamente como "teológicos". El objetivo de este artículo es presentar una interpretación de los motivos y las cuestiones fundamentales que mueven a Hegel en estos primeros escritos, una interpretación que se aleja de planteamientos teológicos y que ve el sentido de la religión para el joven Hegel en íntima conexión con la Revolución Francesa y el republicanismo.

Quizás el problema fundamental desde el que enfocar y entender las reflexiones del joven Hegel sobre religión sea la siguiente pregunta: ¿cómo entender y crear una religión que no sea instrumento de dominación, que no sea una instancia de legitimación para la opresión de unos seres humanos sobre otros, sino un elemento cohesionador de la sociedad en pos de la democracia y la libertad? Esta pregunta tiene en la actualidad al igual que entonces mucha relevancia, tanto para responderla escépticamente como para aceptar el reto que plantea. Es característico del pensamiento de Hegel el intento de pensar la religión en su esencia como espíritu y, con ello, el intento de explicitar una conexión que se pretende fundamental entre religión, por un lado, y libertad y razón, por otro. Con su filosofía, Hegel pretende mostrarnos que se puede y se debe pensar una religión que sea garante y expresión de la razón y la libertad, a pesar del papel fáctico que la instituciones religiosas han jugado dentro las estructuras de poder a lo largo de la historia.

En estas páginas vamos a intentar desarrollar todas las implicaciones de esta cuestión en las primeras reflexiones sobre la religión que hace Hegel en Berna. Se trata de un Hegel recién salido del seminario de Tubinga donde estudió teología, muy influenciado por las lecturas de Kant y Rousseau, que plantea la religión desde un punto de vista muy alejado del de sus profesores teólogos. La idea kantiana de una Iglesia y el concepto de religión civil de Rousseau son los dos ejes en los que se encuadra su primer proyecto eminentemente práctico - de una religión popular. Veremos, igualmente, cómo y por qué abandona Hegel este proyecto y se vuelca en el análisis del fenómeno de la positividad de la religión cristiana, y en qué sentido estos análisis son una continuación de sus preocupaciones sobre religión y republicanismo.

\section{El proyecto de una religión popular}

"Que el fin y la esencia de toda verdadera religión y también de la nuestra es la moralidad de los hombres"

De esta manera caracteriza el joven Hegel en Die Positivität der christlichen Religion el sentido y fin de toda religión, el patrón filosófico, en el cual toda religión ha de medirse: si, en definitiva, contribuye a acrecentar la moralidad de los seres humanos. El fin último de toda religión verdadera no es para el joven Hegel otro que la construcción de una sociedad moral y virtuosa en la que los seres humanos vivan en común con el amor como elemento unificador fundamental. Tal es, sin embargo, en ningún caso un pensamiento original del

${ }^{1}$ Hegel, G.W.F., Werke in zwänzig Bände, Frankfurt am Main, Suhrkamp, 1971, tomo I, p. 105. Traducción mía, original alemán: "Daß der Zweck und das Wesen aller wahren Religion und auch unserer Religion Moralität der Menschen sei”. 
joven Hegel, sino más bien el sentido último que igualmente dio ya Kant a la religión en sus reflexiones, una caracterización puramente kantiana. En efecto, Kant había afirmado claramente que la victoria del bien sobre el mal en el mundo solamente es posible bajo la condición de un proyecto social, la construcción de una sociedad moral, en la que los seres humanos se relacionen entre sí no en términos legal-jurídicos, sino en tanto que seres morales:

"El dominio del principio bueno, en cuanto los hombres pueden contribuir a él, no es, pues, a lo que nosotros entendemos, alcanzable de otro modo que por la erección y extensión de una sociedad según leyes de virtud y por causa de ellas",2.

El problema del mal, por tanto, requiere en términos kantianos una respuesta comunitaria y no puede reducirse a un asunto personal. Y la respuesta a tal problema es para Kant la religión en sentido racional: el proyecto de configuración de una sociedad moral. ${ }^{3}$ Esta concepción de la religión está presente sin lugar a dudas en los primeros escritos hegelianos sobre religión popular. Más aun: se puede caracterizar el pensamiento de Hegel en Berna como la aplicación concreta de la filosofía práctica de Kant. Para Hegel, el concepto kantiano de religión constituye el punto de encuentro entre naturaleza y libertad, la clave para la realización de la ley moral en el mundo sensible. Y su primer pensamiento consiste, por ello, en la puesta en práctica de ese proyecto religioso de traer moralidad a los hombres.

Sin embargo, Hegel redefine desde el principio las ideas kantianas. Pues para él, el concepto filosófico de religión esbozado por Kant ha de verse realizado bajo la forma de una religión popular. El tema de la religión popular era un tema candente a finales del siglo XVIII entre los seguidores de la Revolución Francesa. Tal programa estaba entonces de plena actualidad debido a los intentos de Robespierre de encontrar nuevas formas de culto religioso y patriótico. La idea procede del final del Contrato social de Rousseau bajo el nombre de "religión civil". ${ }^{4}$ Se trataba, en definitiva, para Rousseau y para los revolucionarios franceses, de conseguir que la religión dejase de ser una instancia ideológica en pos de la opresión del pueblo y se convirtiese en la valedora de los principios republicanos: una religión de la libertad, la igualdad y la fraternidad, donde Dios no aparezca como Señor, sino como garante de la libertad de los ciudadanos. El hecho de que los primeros escritos de Hegel se ocupen de la religión no responde, por tanto, a una posición reaccionaria en contra de las nuevas tendencias revolucionarias de la época, sino al convencimiento de que también en una república libre, y no en menor medida en pos de ella, la religión puede y debe tener cabida como una de las ocupaciones más importantes de nuestras vidas. ${ }^{5}$ La concepción de una religión libre del despotismo estaba, sin embargo, todavía por formular, y ésa es la tarea que Hegel se pone a sí mismo, con la esperanza de que las tendencias revolucionarias triunfen también en su tierra, Württemberg. ${ }^{6}$

${ }^{2}$ Kant, I., La religión dentro de los límites de la mera razón, Madrid, Alianza, 2006, p. 94.

${ }^{3}$ En este sentido no podemos estar de acuerdo con G. Lúkacs cuando éste sitúa la diferencia entre Kant y Hegel a este respecto en el hecho de que Hegel, supuestamente a diferencia de Kant, sitúe a la praxis de la sociedad desde el principio en el centro de su pensamiento. Muy al contrario, el mismo Kant había ya colocado a la colectividad en el centro de la cuestión. Cfr. Lúkacs, G., "Der junge Hegel. Über die Beziehungen von Dialektik und Ökonomie", en: Lúkacs, G., Werke, Zürich y Berlin, Luchterhand, 1967, tomo VIII, p. 40.

${ }^{4}$ Rousseau, J. J., El contrato social, libro IV, cap. VIII.

5 "Eine der wichtigsten Angelegenheiten unseres Lebens". Hegel, G.W.F., Werke I, op. cit., p. 9.

6 Cfr. a este respecto también el artículo de Hans Friedrich Fulda, "Rousseausche Probleme in Hegels 
El joven Hegel ve el concepto kantiano de religión y el programa de religión popular de Rousseau intrínsecamente conectados. La religión de la razón ha de ser igualmente religión del pueblo, y para ello, para que sea real, Hegel se ve obligado a discutir ciertos puntos de la idea kantiana de una Iglesia. Pues si la religión, en su proyecto moral, ha de tener efecto, entonces es necesario rebajar su concepto puro al plano de la fantasía, de la sensibilidad, en definitiva, al plano del pueblo. Precisamente porque Hegel se plantea en todas sus consecuencias la tarea de pensar una religión racional que sea real y conecte con la gente, es por lo que tiene las mayores discrepancias con las características de una religión racional dadas por Kant. Tanto, que de los cuatro principios de una Iglesia en sentido kantiano ${ }^{7}$ sólo uno queda en pie. Recordemos esos cuatro principios fundamentales de una religión racional según Kant: universalidad, pureza, libertad-democracia e inmutabilidad. Por universalidad entiende Kant en este contexto la aspiración de una Iglesia racional de dotar a sus doctrinas de una validez universal y de unificar a toda la Humanidad bajo ella, pues tal Iglesia funda sus enseñanzas y prácticas meramente en la razón humana. Esta idea está como mínimo puesta en cuestión por Hegel en Berna, sobre todo en die Positivität der christlichen Religion, donde las tendencias universalistas y expansionistas de la Iglesia cristiana son juzgadas siempre de forma negativa. El objetivo kantiano de fundar una única religión universal no es compartido por el joven Hegel. Para él es aceptable e incluso deseable una pluralidad de religiones, siempre que estas se concentren en lo esencial: virtud, amor y pureza de corazón. Porque cómo ha de conseguirse esto, es un problema que no puede ser resuelto en abstracto, sino que requiere una consideración concreta del espíritu de cada pueblo y cada época. Esta convicción de Hegel está en conexión, bajo nuestro punto de vista, con el hecho de que en el programa de una religión popular tanto pureza como inmutabilidad se vean igualmente relativizadas. Ambos aspectos significan para Kant que, por un lado, la religión racional sólo debe admitir sentimientos y emociones moralmente puros, y por otro lado, que sólo puede contener principios que se deriven a priori de la moralidad. Esto significa que, para Kant, quedan excluidos de una religión racional toda superstición, todo símbolo arbitrario y toda fantasía. Para Hegel, al contrario, precisamente la mezcla adecuada entre una doctrina racional y la fantasía y sensibilidad juega un papel fundamental en la religión popular. Tanto las doctrinas religiosas basadas en la razón pura como los usos y rituales deben adecuarse al espíritu de cada pueblo y emanar de él. El joven Hegel anhela una religión que brote de la realidad de los hombres y mujeres, de su sufrimiento, sus preocupaciones, sus sentimientos y su vida, y que con libertad y autonomía construya un estado moral (moralischer Zustand) entre ellos. En el fondo de estos pensamientos se encuentra, sin duda, una cada vez más creciente crítica al dualismo kantiano en el ser humano.

Así, el proyecto del joven Hegel puede resumirse en una fórmula: reordenar el presente moralmente. En este punto, su posición se diferencia de las ideas ilustradas y de Kant, en la medida en que para Hegel no se trata de contraponer tendencias opuestas a la realidad cotidiana del pueblo, sino de apoyarse en las costumbres, fiestas y prácticas de la gente común y redirigirlas en sentido moral sin tener que eliminarlas o censurarlas por completo. Por ello, Hegel resume lo principal de una religión popular de la siguiente manera: combinar en una comunidad humana viva los principios de la razón pura práctica con la especifidad del corazón y la fantasía del pueblo, una comunidad, en la que realmente toda la

Entwicklung", en Fulda, H. F. y Horstmann, R-P. (ed.), Rousseau, die Revolution und der junge Hegel, Stuttgart, Klett-Cotta, 1991, p. 47.

${ }^{7}$ Cfr. Kant, I., La religión, op. cit., p. 102. 
actividad de la vida pública encuentre su seno. Intenciones dogmáticas o paternalistas no tienen cabida en tal proyecto, pues buscan más la vanidad y la soberbia que la mejora real del corazón de los hombres.

Sigamos profundizando en el concepto de religión que maneja el joven Hegel. En sus Fragmenten über Volksreligion und Christentum encontramos una distinción capital para entender la problemática que él intenta plantear, a saber, la distinción entre religión en sentido subjetivo y en sentido objetivo. Esta distinción no es original de Hegel, sino que proviene de la teología de la época. ${ }^{8}$ Para los teólogos de entonces, la religión objetiva trataba de la doctrina y los dogmas de fe del cristianismo. La religión subjetiva, en cambio, se ocupaba de sentimientos, de educar moralmente, de hacer que la religión tenga en general efecto sobre la vida de los creyentes. Ya según esta teología de la época el principal objetivo de la religión subjetiva consistía en encauzar las tendencias e instintos más naturales de los seres humanos y enfocarlos hacia la moralidad y el bien. La clave a éste respecto para entender al joven Hegel es que para éste el verdadero concepto de religión se identifica exclusivamente con lo que los teólogos de la época denominaban religión subjetiva, mientras que para él la llamada religión objetiva no supone más que fórmulas muertas y vacías de todo espíritu y toda moralidad que han de memorizarse, pero que no tienen en el fondo nada que ver con la religión en sentido propio. El siguiente texto es meridianamente claro al respecto:

"Pues la religión es una cuestión del corazón, el cual a menudo actúa de manera inconsecuente con los dogmas que su entendimiento o su memoria adopta; los hombres más dignos de respeto no son seguro siempre aquellos que más han especulado sobre religión, aquellos que convierten su religión muy a menudo en teología, esto es, que cambian habitualmente la plenitud y cordialidad de la fe por fríos conocimientos y sentencias.

Al contrario, la religión gana muy poco con el entendimiento, sus operaciones y sus dudas pueden, al contrario, enfriar más el corazón que calentarlo."9

Desde el punto de vista del joven Hegel, la religión objetiva es, como vemos, una masa de dogmas, de argumentos vacíos, de discursos e investigaciones sobre temas baladíes, un edificio teórico que la imaginación y la palabrería elevan al estatus de un palacio de la ciencia, pero que no aporta a las personas ni un átomo de religiosidad. Tales elucubraciones no tienen, en efecto, ninguna consecuencia en lo que a la moralidad o inmoralidad de los seres humanos se refiere, porque se basan en memoria y entendimiento, y sobre todo del entendimiento no cabe esperar nada respecto a la mejora real del alma humana. Si el entendimiento no puede ayudar a los hombres en la tarea de educar en valores, en sentimientos honestos y en autonomía y pureza de espíritu, tampoco lo hará un subproducto de él, la religión en sentido objetivo.

\footnotetext{
${ }^{8}$ Cfr. Schmidt, T. M.: „Religion und Sittlichkeit in Hegels Frühschriften“, en Bondeli, M. (ed), Hegels Denkentwicklung in der Berner und Frankfurter Zeit, Munich, Fink, 1999, pp. 225-249.

${ }^{9}$ Hegel, G.W.F., Werke I, op. cit., p. 19. Traducción mía, original alemán: "denn Religion ist Sache des Herzens, welches oft inkonsequent handelt gegen die Dogmen, die sein Verstand oder Gdächtnis annimmt; die verehrungswürdigsten Menschen sind gewiß nicht immer diejenigen, die am meisten über Religion spekuliert haben, die ihre Religion sehr oft in Theologie verwandeln, d. h. oft Fülle, Herzlichkeit des Glaubens gegen kalte Erkenntnisse und Wortparaden vertauschen. Religion gewinnt durch den Verstand aber sehr wenig, seine Operationen, seine Zweifel können mi Gegenteil das Herz mehr erkalten als wärmen.”
} 
"Pero tal y como la facultad de este entendimiento no tiene un gran momento cuando se trata de conseguir la mejora de los hombres, de conseguir una educación en disposiciones morales mayores y más fuertes, en sentimientos nobles, en una autonomía decidida, tampoco tiene el producto del entendimiento, la religión objetiva, gran importancia en tal empresa". ${ }^{10}$

Como vemos, la religión objetiva es para el joven Hegel una deriva ilusoria que no aporta nada al verdadero objeto de la religión. Sin embargo, lo que al principio de los fragmentos aparece como elucubraciones vacías que, si bien desvían a la religión de su verdadero fin, parecen a primera vista inofensivas, va paulatinamente tomando un cariz más negativo. Así, Hegel va subrayando cada vez más en sus textos el carácter perverso y despótico de esta religión objetiva, y la idea de la objetividad en la religión se va desarrollando poco a poco hasta acabar en la idea de la positividad de la religión, un concepto central en la etapa de Berna. La primacía de la religión objetiva entronca para Hegel con la creencia en la tradición y con una praxis artificialmente reglada que se centra en la repetición de rituales y dogmas en vez de en el punto central de la religión: la construcción de una comunidad fraternal unida por el amor y la moralidad como principios fundamentales. Toda esa estructura, en sus tres perfiles, el discursivo-doctrinario, el perfil práctico de ritos y ceremonias vacías y el lado político-social, con el poder institucional de la Iglesia, va a ser entendida por el joven Hegel paulatinamente bajo un nuevo concepto, el de positividad, para designar el factor común y fundamental a todo ello: la separación de la religión del corazón de los hombres y de la vida del pueblo y su posición como elemento externo a ellos, elemento que más que surgir de ellos, se les opone y les oprime.

\section{Religión y república}

En el tratamiento que hace el joven Hegel de la religión destacan principalmente dos problemas fundamentales que se plantean desde el principio. Solamente el carácter de ambas cuestiones pone de manifiesto hasta qué punto Hegel se toma en serio el proyecto de una religión racional. El primer problema, que podemos rastrear en los Fragmenten über Volksreligion und Christentum (1793 - 1794), consiste en definir lo más claramente posible la forma concreta que la Iglesia ha de tomar desde un punto de vista racional. Como hemos visto, Hegel va más allá de las consideraciones generales de Kant sobre la idea de una Iglesia y concentra su interés en formular un programa exhaustivo dirigido ya no solamente a intelectuales y filósofos, sino al público en general. En este sentido discute sobre todo cuestiones puramente prácticas y muy concretas de una - futura - religión racional: qué tipo de ceremonias han de oficiarse, qué preceptos han de seguirse, qué pasiones y sentimientos han de fomentarse y alabarse, y cuáles han de ser censurados, o, por ejemplo, hasta qué punto es la superstición tolerable o inevitable.

Pero en sus consideraciones prácticas se plantea en el pensamiento del joven Hegel inevitablemente también una segunda cuestión, a saber, hasta qué punto se adecua el cristianismo, la religión existente, a la idea de una Iglesia en sentido racional. Si bien Hegel, por tanto, piensa dentro del marco de la filosofía de la religión de Kant, él es mucho más radical que éste, en la medida en que toma la idea de una religión racional como

\footnotetext{
10 Ibid., p. 27. Traducción mía, original alemán: “Aber wie die Kraft [dieses Verstandes] kein großes Moment hat, wenn Besserung der Menschen, Auferziehung zu großen starken Gesinnungen, zu edlen Gefühlen, zu einer entschlossenen Selbständigkeit hervorgebracht werden soll, so hat auch das Produkt, die objektive Religion, kein großes Gewicht dabei."
} 
criterio evaluador y examina a la religión cristiana y a la Iglesia de la época. Su pensamiento, no obstante, no llega a ser nunca anticristiano. Lo que a Hegel le interesa más bien es dilucidar, en qué medida la religión cristiana constituye una realización de la idea de una religión racional, y a causa de qué factores la Iglesia de Jesús no se corresponde del todo con el ideal a alcanzar. La idea de fondo es, sin duda, que sólo la comprensión de las carencias de la religión de su tiempo permitirá una reforma de ésta a mejor.

La primera de éstas dos cuestiones es tratada en los Fragmenten über Volksreligion und Christentum, la segunda, sobre todo, en Die Positivität der christlichen Religion. Si bien es cierto que, como ya hemos mencionado, Hegel se mueve continuamente dentro del marco de la filosofía kantiana, no es menos cierto que discute internamente con ella en algunas cuestiones. La causa de ello es, sobre todo, que la concreción hegeliana de la fillosofía práctica de Kant trae consigo como consecuencia, debido a su radicalidad, que los pensamientos de Kant se vean en el proceso parcialmente modificados.

En el primer punto hemos visto ya las importantes modificaciones que Hegel lleva a cabo respecto a la concepción kantiana dentro de su programa de una religión popular. La influencia de Rousseau y de la Revolución Francesa son aquí determinantes. Pero queda otra diferencia fundamental por tratar, una diferencia que está en conexión precisamente con la radicalidad del planteamiento hegeliano. Se trata de la conexión que Hegel establece, al igual que Schiller y, después de éste, Hölderlin, entre la filosofía de la religión de Kant y la religión popular en la antigua Grecia. Hegel iguala la idea de una religión racional expuesta en el Religionsschrift de Kant con la imagen histórica y fuertemente idealizada de la religión en la antigua Grecia, de manera que compara la realidad de su tiempo, por un lado, con la idea de una sociedad moral que se rija según principios éticos, y por otro, con la antigua Grecia, que figura como un modelo a alcanzar, como el pasado originario y perdido. Desde nuestro punto de vista este hecho tiene mucho que ver con la intención y concepción de Hegel en estos escritos. A Hegel no le basta la doctrina kantiana de los postulados, pues aspira a vivir un verdadero triunfo de la moralidad en su realidad más actual y cercana, y por ello busca en la historia una época en la cual la anhelada comunidad de la virtud fuera real. Por este motivo se refiere tantas veces a la antigua Grecia, igual que a la vida de Jesús y su comunidad de discípulos. Hegel se interesa por las causas del hundimiento de estas comunidades ideales y mantiene siempre de fondo la pregunta acerca de cómo se puede recuperar nuevamente este ideal. Lúkacs ya planteó con mucha razón que la oposición entre moralidad y realidad, o entre el mundo inteligible de la libertad y el mundo sensible, posee en el joven Hegel, a diferencia de en Kant, un carácter histórico. Efectivamente, esta oposición es para Hegel la característica principal de su tiempo, un tiempo que tiene su ejemplo contrapuesto en la verdadera unificación de moralidad y realidad en el momento histórico de la antigua Grecia. Tal historización del dualismo kantiano explica, en efecto, la esperanza de Hegel de una nueva superación real del mismo. $^{11}$

A partir de 1974 el programa de una religión popular no tiene continuidad en Hegel, lo cual coincide históricamente con la caída de Robespierre. Las esperanzas de que la Revolución se extienda a Württemberg se desvanecen y el proyecto de la religion civile pierde con la caída de los jacobinos su actualidad. Además, las noticias que llegan desde Francia sobre el terror que rodea al proceso revolucionario traen consigo una decepción en muchos intelectuales sobre la Revolución Francesa, también en Hegel. Todo esto explica el abandono de la tarea de formular un programa para una religión popular. A partir de

${ }^{11}$ Cfr. Lúkacs, G., Der junge Hegel, op. cit., p. 57. 
entonces, Hegel se va concentrar en investigar las causas del surgimiento del despotismo y de lo que el llama "positividad" en la religión cristiana. Sus preocupaciones, sin embargo, siguen siendo muy prácticas y siguen estando alejadas de temas especulativos. Die Positivität der christlichen Religion no supone en este sentido ningún cambio en en el pensamiento de Hegel, que sigue estando fuertemente conectado con la realidad social de su tiempo. El resultado principal de la crítica hegeliana a la positividad de la religión cristiana consistirá en que la corrupción de la religión y su deriva hacia la positividad ha de entenderse como una ruptura entre la subjetividad viva del pueblo y las formas doctrinales y materiales de la Iglesia. Es, para el joven Hegel, ésta ruptura entre la realidad viva del pueblo y la Iglesia, la causa fundamental de la positividad que caracteriza a la religión cristiana.

La investigación sobre los orígenes de la positividad de la religión y el consecuente despotismo en la Iglesia se enmarca dentro de una constatación para Hegel: la conexión manifiesta entre la Iglesia de su época y el absolutismo, como queda claro a raíz de una carta dirigida a Schelling el 16 de abril de $1795 .{ }^{12}$ Ambas instituciones, escribe Hegel a Schelling, comparten intereses y se apoyan mutuamente. No sería una exageración afirmar que el joven Hegel caracteriza en esta carta a la iglesia oficial como una institución ideológica. La religión oficial y sus instituciones tienen como objeto principal de sus enseñanzas la justificación del despotismo. Se trata, con ello, de un discurso legitimador del status quo: en la medida en que el discurso religioso insiste continuamente en cómo deberían ser los seres humanos y cuán pecaminosos en realidad son, remarca constantemente esta diferencia, proyecta cualquier esperanza de un mundo mejor fuera del terreno de lo posible y elimina con ello toda iniciativa de cambio político. La Iglesia persigue, por tanto, no la educación moral de las personas, sino el bloqueo de toda crítica al presente y de toda aspiración de mejora social. El punto central para ello es la postulación de un deber-ser irrealizable, el cual, precisamente porque se define en contraposición con la baja naturaleza humana, elimina toda esperanza realista de progreso en la sociedad. La Iglesia fomenta así la idea de que ante el mundo y las estructuras sociales de poder no cabe más que resignarse, pues por parte de los seres humanos no cabe esperar ninguna mejora.

Esta carta, entre otros fragmentos, nos muestra hasta qué punto la posición de Hegel está lejos de una consideración abstracta e ingenua de la religión. Cuando Hegel escribe su crítica a la religión cristiana, está ahí contenida no sólo la idea de otra religión sino, dada la alianza entre religión positiva y despotismo de la que Hegel es consciente, también la idea de otra sociedad. La concepción kantiana de una religión racional ya ponía como condición la existencia de una república de ciudadanos libres, que debía servir de base para una Iglesia en sentido racional. Esta idea es sin duda retomada y ampliada por el joven Hegel. En el fondo de sus reflexiones está el convencimiento de que verdadera religión y república están esencialmente conectadas, de manera que ni religión sin república, ni república sin religión son deseables. El proyecto religioso, de esta manera, no puede entenderse sin un respectivo proyecto político:

\footnotetext{
"Espíritu del pueblo, historia, religión, nivel de libertad política del mismo están entrelazados en un nudo, y no pueden ser tratados por separado ni en cuanto a su naturaleza ni en cuanto a su influencia entre sí [...]. Formar la moralidad de un hombre concreto es tarea de la religión privada, de los padres, del propio es fuerzo y de las circunstancias; formar el espíritu de un pueblo es en parte tarea de la religión popular, en parte de las condiciones políticas."13

${ }^{12}$ Briefe von und an Hegel, hrsg. von Johannes Hoffmeister, tomo I, Hamburgo, Felix Meiner, 1969, p. 24.

${ }^{13}$ Hegel, G.W.F., Werke I, op. cit., p. 42. Traducción mía, original alemán: „Geist des Volks, Geschichte,
} 
Verdadera religión no puede surgir para Hegel de otro sitio que de la libertad del pueblo, por lo que el programa religioso en sentido racional, a saber, traer moralidad a los hombres, sólo puede llevarse a cabo dentro el marco de un proyecto republicano. A la crítica a la religión y su positividad ha de acompañar por tanto la crítica a la relación existente entre la Iglesia positivo-despótica y el Estado absolutista del Antiguo Régimen. Esta crítica política se encuentra sobre todo al final así como en los añadidos (Zusätze) del Positivitätsschrift. De igual manera que en la antigua polis libertad política y religión iban de la mano, también existe para Hegel una conexión entre despotismo político y religioso.

La vida del antiguo ciudadano griego o romano era para Hegel una vida pública, una vida que unificaba política y religión. Los ciudadanos libres sólo obedecían las leyes que ellos se daban a sí mismos; su religión era igualmente una religión de la virtud: ni aprendían ni imponían reglas a nadie, sino que practicaban sus máximas morales mediante sus acciones. Su república era su bien más preciado, expresión de su libertad y del espíritu de su pueblo, y por ella estaban dispuestos a darlo todo, tanto sus posesiones como, si fuera necesario, su vida. En efecto, la disposición a dar su vida por el bien común es para Hegel una de las diferencias más fundamentales entre el ideal de las antiguas Grecia y Roma y la sociedad de su presente.

Con el surgimiento de la positividad tanto religiosa como política ${ }^{14}$ desaparecen estos ideales. El hombre abandona la imagen del Estado como producto de su propia acción y su libertad; ya no se reconoce más a sí mismo en él. El Estado se convierte en una estructura ajena para él, en la que su voluntad no juega ningún papel. Su religión, el cristianismo positivo, ya no busca formar y fomentar una comunidad en el amor, sino que se vuelca cada vez más en el individuo: su doctrina consiste en aislar al ser humano y mostrarle su imperfección, su pecado y su distancia respecto al ideal santo. El único efecto buscado en el individuo por parte de la religión positiva es la culpa, que hace que éste se concentre cada vez más en sí mismo. A la positividad de la religión le corresponde, según el joven Hegel, un fenómeno paralelo en la vida política. Pues despotismo religioso y despotismo político tienen su raíz en la misma pérdida de libertad por parte del pueblo. La esclavitud del hombre tiene, por tanto, dos caras: por un lado, la positividad de la religión, por otro lado, el poder estatal ajeno, un poder en el que el pueblo ni se siente representado ni puede reconocerse. $\mathrm{Y}$ de igual manera, su liberación política, democrática, está irrenunciablemente unida con la recuperación y el cultivo de los ideales y virtudes éticas y políticas, una tarea que, para el joven Hegel, es esencialmente religiosa.

\footnotetext{
Religion, Grad der politischen Freiheit desselben lassen sich weder nach ihrem Einfluß aufeinander, noch nach ihrer Beschaffenheit abgesondert betrachten, sie sind in ein Band zusammenverflochten [...] Die Moralität einzelner Menschen zu bilden, ist Sache einer Privatreligion, der Eltern, eigener Anstrengung und der Umstände; den Geist des Volks zu bilden, ist zum Teil auch Sache der Volksreligion, zum Teil der politischen Verhältnisse."

${ }^{14}$ Hegel no utiliza nunca la expresión "positividad política". Sin embargo, dada la analogía entre el fenómeno de positividad religiosa, donde una institución religiosa se erige autoritariamente frente a la subjetividad del pueblo, sin que éste pueda reconocerse en ella, y el correspondiente fenómeno político, donde el mismo pueblo no se reconoce en sus leyes, sino que éstas tienen su origen en un aparato estatal extraño, creo que el usto de tal expresión está suficientemente fundado.
} 


\section{Reflexiones finales}

Hemos seguido la evolución de Hegel en torno al problema de la religión en Berna. Hemos visto como el joven Hegel está influenciado en su pensamiento fuertemente por Rousseau y, sobre todo, por la filosofía práctica kantiana. La concepción kantiana de la religión es retomada por él con entusiasmo como el punto clave con vistas a la realización de la ley moral en el mundo sensible. Sin embargo, hemos visto igualmente cómo en el desarrollo de éste kantiano radical está presente desde el principio la discusión con Kant. Éste desarrollo es sin duda característico de Hegel, es algo que se repite a lo largo de todo su pensamiento: la necesidad de criticar a Kant, de ir más allá de él, precisamente en la medida en que Hegel toma en serio y desarrolla el pensamiento kantiano hasta sus últimas consecuencias.

Si dejamos ahora a un lado el proyecto de una religión popular, abandonado por el propio Hegel, podemos decir que el concepto de positividad es sin duda el concepto central en las reflexiones sobre religión en Berna. La positividad en todas sus formas - religiosa y política - lleva al ser humano en última instancia al individualismo. Su libertad no encuentra ni en el estado ni en la religión seno alguno. En la Antigüedad, la libertad de los ciudadanos tenía su realización en la República, pero en la sociedad moderna toda actividad y todo fin del hombre se encuentra reducido a lo individual. La libertad sólo puede verse realizada en la propiedad, y toda ley y todo derecho tienen un objetivo: asegurar la propiedad privada.

Estas constataciones abren sin duda una encrucijada en el pensamiento del joven Hegel. Por un lado, Hegel es ya entonces un decidido republicano. Es un decidido defensor de una sociedad libre y democrática, en la que el antiguo siervo entre como ciudadano de pleno derecho. Por otro lado, también reconoce desde muy temprano la tendencia de la sociedad burguesa a girar entorno a la propiedad privada y hacer depender todo de ella. El gran avance en libertad que supone la derrota del absolutismo y el establecimiento de una república democrática puede perder pronto todo su sentido, si la libertad conquistada acaba reduciéndose a la propiedad privada. Y éste es un problema que, en referencia a la posterior filosofía del espíritu objetivo, no conviene perder de vista. 